\title{
Integración y exclusión de inmigrantes colombianos recientes en Santiago de Chile: estrato socioeconómico y "raza" en la geocultura del sistema-mundo"
}

\author{
Emiliano Nicolás Gissi-Barbieri y Gonzalo Ghio-Suárez
}

\author{
Universidad de Chile, Chile
}

\begin{abstract}
Resumen
El artículo explora el proceso de integración de los inmigrantes colombianos en Santiago de Chile. La conclusión es que hay importantes diferencias en la integración de los diferentes grupos socioeconómicos y "raciales" de inmigrantes, particularmente los inmigrantes afrodescendientes se ven expuestos a situaciones de discriminación racial. Dado que en Chile la población afrodescendiente es de reciente llegada, el racismo hacia ellos debe explicarse contextualizando la "cultura nacional" en la geocultura del sistema-mundo, que contiene una jerarquización racial conformada en la época colonial para dar fundamento a su división racial del trabajo. En este marco son recibidos los inmigrantes en Chile, país imbuido en esta geocultura colonialista y eurocéntrica que explica el trato diferenciado dado a los distintos colectivos de inmigrantes.
\end{abstract}

Palabras clave: Inmigrante, integración, racismo, sistema-mundo, geocultura.

Abstract

Integration and exclusion of recent colombian immigrants in Santiago de Chile: socioeconomic stratum and 'race' in the geoculture of world-system

The article explores the process of integration of Colombian immigrants in Santiago of Chile. The conclusion is that there are important differences in the integration of the different socioeconomic and "racial" groups of immigrants, in particular afrodescendant immigrants are exposed to situations of racial discrimination. Given that the afrodescendant population in Chile is newly arrived, racism against them must be explained contextualizing the "national culture" in the geoculture of the world-system, that contains a racial hierarchy shaped in the colonial era to support its racial division of labor. In this context, immigrants are received in Chile, a country imbued in this colonialist and eurocentric geoculture that explains the differential treatment given to the different immigrant groups.

Key words: Immigrant, integration, racism, world-system, geoculture.

* El siguiente artículo presenta resultados del proyecto Fondecyt $\mathrm{N}^{\circ} 11130287$, programa al cual
expresamos nuestros agradecimientos. 


\section{INTRODUCCIÓN}

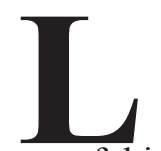

as formas como son tratados los diferentes colectivos de inmigrantes se fundamentan en patrones culturales que se encuentran previamente entre la población del país de recepción. La xenofobia o xenofilia hacia los inmigrantes está típicamente asociada a prejuicios basados en la historia colonial y neo-colonial. Hace dos décadas Chile comenzó a recibir flujos migratorios significativos, y desde el año 2010 en estos flujos comenzaron a tener una presencia notoria los colectivos de países latinoamericanos con población afrodescendiente. En la sociedad chilena no hay una presencia histórica significativa de población afrodescendiente, ${ }^{1}$ y los estereotipos racistas del país se han dirigido principalmente a las personas pertenecientes a los pueblos originarios, y a mestizos con características fenotípicas de dichas etnias. Por estos motivos, un trato discriminatorio hacia los/as inmigrantes afrodescendientes no se podría comprender en base puramente a una "cultura nacional", sin considerar su vinculación a los patrones racistas que circulan en el sistema-mundo.

El presente artículo plantea que a través del análisis de las dinámicas de integración y exclusión de los inmigrantes colombianos en Santiago, se puede apreciar cómo las formas discriminatorias chilenas se corresponden con las modalidades racistas de la geocultura del sistema-mundo capitalista descritos desde la Perspectiva Decolonial, mostrando así que el sistemamundo contiene una geocultura general que se concretiza en cada país y región a través del "colonialismo interno" (González Casanova, 2006), donde se tiende a hipervalorar a la población "blanca" de origen europeo y estadounidense y a minusvalorar a la población "negra" e indígena (como lo expresaran las obras clásicas de Palacios Raza Chilena, en 1904, y de Bonfil Batalla México Profundo, en 1987; este último desde una perspectiva crítica). Así, ocurre que los colombianos afrodescendientes se escapan del racismo intra-colombiano (donde ocupan el estrato más bajo de la población) para buscar nuevas oportunidades en una sociedad chilena que también se vivencia como racista.

A partir del incremento en la inmigración que ha recibido Chile durante las dos últimas décadas y principalmente en los últimos años, se han retematizado públicamente los fenómenos de la integración, la discrimina-

\footnotetext{
${ }^{1}$ La excepción es la Región de Arica y Parinacota, en el extremo norte del país, donde habría 4.7 por ciento de población que se reconoce como afrodescendiente (INE, 2014).
} 
ción y la seguridad pública en relación a los inmigrantes. ${ }^{2}$ Incluso en la actualidad se ha producido una inédita politización de la inmigración, ya que en el contexto de la carrera presidencial por las elecciones que habrá a fines del presente año, diferentes candidatos han planteado el tema de la inmigración como un problema, vinculándolo a supuestos riesgos de seguridad pública. ${ }^{3}$ La exclusión social de los inmigrantes es un factor que afecta la convivencia social entre los colectivos foráneos y los locales. Así, partimos de la premisa que la inclusión de los distintos sujetos inmigrantes es un bien social y cultural, con incidencias positivas sobre la calidad de la convivencia en los diversos espacios sociales.

Este artículo describe las formas y dinámicas de integración de los inmigrantes colombianos/as en la Región Metropolitana de Chile, analizando sus patrones de integración, exclusión y discriminación, tomando la Perspectiva Decolonial de la geocultura del sistema-mundo como marco explicativo de las formas que adquiere el trato racista chileno hacia algunos grupos de inmigrantes. El interés en el colectivo colombiano surge dado que tiene una heterogeneidad que no se encuentra en otras comunidades de inmigrantes, en términos de su pluralidad "racial" y socioeconómica, lo que permite analizar cómo la cultura racista afecta de manera diferenciada a personas que provienen de un mismo país pero que tienen disímiles condiciones socioeconómicas y apariencia fenotípica o "racial".

La metodología utilizada parte por realizar una caracterización general del colectivo colombiano a través de la información que entregan las bases de datos del Departamento de Extranjería y Migración del Ministerio del Interior y Seguridad Pública (en adelante DEM). Luego se analiza el proceso de integración y sus dificultades a partir de una base de datos con más de cincuenta entrevistas en profundidad hechas a inmigrantes colombianos y colombianas residentes en la Región Metropolitana. A partir del trabajo de campo realizado entre los años 2014 y 2015 en las comunas de

\footnotetext{
${ }^{2}$ Por ejemplo, el pasado 30 de Agosto de 2016 "El Mercurio", probablemente el diario de mayor influencia del país, de orientación conservadora-neoliberal, publicó como principal titular de su primera plana "El 52 por ciento de los imputados por delitos en el extremo norte del país son ciudadanos extranjeros". En la noticia publicada en la sexta página del cuerpo C, se puede comprobar que por "extremo norte" el periódico sólo se refiere a la región de Arica y Parinacota, y que la mayor parte de los delitos de los que se dan cuenta son "delitos transnacionales", que incluyen actos que sólo pueden realizar extranjeros, como el cruce clandestino de la frontera por pasos no habilitados, el no poseer documentos legales, etc., o crímenes que por su carácter transnacional es muy probable que en una zona de frontera sean cometidos por chilenos y ciudadanos de diferentes países, como el tráfico de inmigrantes, la trata de personas o el tráfico de drogas.

${ }^{3}$ Principalmente han sido los precandidatos de la coalición de derecha "Chile Vamos". Véase por ejemplo: http://www.elmostrador.cl/noticias/pais/2016/11/28/ossandon-endurece-su-discurso-hacia-los-extranjeros-las-puertas-del-pais-como-las-de-la-casa-se-abren-pero-no-a-todos/ y http://www.elmostrador.cl/noticias/pais/2016/11/29/inmigrantes-pinera-le-quita-el-tema-a-ossandon-y-logra-sacar-bancard-de-la-agenda/.
} 
Santiago-Centro, Recoleta y Quilicura, Santiago, se realizaron entrevistas en profundidad a mujeres y hombres de nacionalidad colombiana, quienes al momento de la entrevista tenían entre tres y diez años de residencia en el país ${ }^{4}$. Estas comunas fueron seleccionadas para dar cuenta etnográficamente de la heterogeneidad social y urbana de la capital, respectivamente centro, peri-centro y periferia, espacios en los que se están desarrollando nuevas formas de segregación y mezcla social, debido a la modificación en la escala de la segregación social durante la última década. En el análisis de las entrevistas se buscó distinguir las características del proceso de integración en base a los diferentes estratos socioeconómicos y a las distintas "razas" de los inmigrantes.

Como conclusión general se puede señalar que efectivamente hay diferencias importantes en los procesos de integración según estratos socioeconómicos, y en particular según el color de piel o las "razas" de los inmigrantes. Se constata en las entrevistas que en la mayor parte de los casos los residentes colombianos se integran a los diferentes ámbitos de la sociedad chilena. Esta situación general positiva debe matizarse al considerar los estratos socioeconómicos, pues en los estratos medios-altos y altos se aprecia un mejor proceso de integración que en los medios-bajos y bajos. Sin embargo, la principal distinción debe introducirse sobre los inmigrantes afrodescendientes, quienes en distintas instancias, como el trabajo, los espacios públicos, los transportes urbanos y los servicios, se ven expuestos a situaciones de discriminación e incluso hostigamiento, las cuales necesariamente afectan su proceso de integración, por ejemplo, al evitar el uso del transporte público para sus desplazamientos por la ciudad o al restringir los trabajos a los que pueden acceder. Esta aparición, o explicitación, en los patrones culturales chilenos del racismo hacia los afro-descendientes (Tijoux, 2016), da cuenta de la fuerza que posee la geocultura del sistemamundo en un país con poca población afrodescendiente y que tradicionalmente se ha considerado como "aislado". En Chile esta situación abre una problemática que va más allá de la inmigración colombiana, por la creciente llegada de personas afrodescendientes de países como Haití, República Dominicana, Cuba, Brasil y Ecuador, entre otros.

\footnotetext{
${ }^{4}$ Para realizar este análisis cualitativo los participantes relataron cómo fue su experiencia de preparación del viaje, su llegada y estadía en la sociedad chilena, cuáles fueron aquellas cosas que los descolocaron, aquello positivo, negativo o extraño de su vivencia individual, así como su percepción sobre el país antes y después de migrar y su participación en actividades sociales en Chile. El contenido de las entrevistas fue analizado por medio de una malla temática que se construyó a partir de la pauta de entrevista. El proceso de codificación se realizó paralelamente al de categorización, incluyendo categorías emergentes. Finalmente, se utilizó el software Atlas-ti 7.0 , el que permite visualizar patrones y difundir los resultados.
} 


\section{Planteamiento del problema}

Desde la última década del siglo XX y hasta el presente, se ha constatado un incremento considerable en los flujos migratorios globales e intrarregionales (OIM, 2015). A pesar de que los migrantes representan sólo 3.2 por ciento de la población total en el mundo, su relevancia política y económica ha aumentado en las últimas décadas, particularmente en regiones centrales del sistema-mundo como Europa y Estados Unidos, donde inmigrantes y refugiados han pasado a ser un tema central de las campañas políticas de los últimos años, y discursos anti-migrantes han incidido en resultados de elecciones, como la de Donald Trump en Estados Unidos y en el triunfo del "Brexit" en el Reino Unido. Esto ha dado pie a cambios en la legislación migratoria de los países históricamente receptores, como Estados Unidos y España, proclives a un mayor control migratorio (Sassen, 2015; Portes, 2012). Así, no sólo han crecido las corrientes migratorias en cuanto a su tamaño y diversidad, sino que se ha convertido en el centro de debates políticos, en términos de su efecto sobre el empleo y el desarrollo de los países, en relación a los derechos humanos, sobre los procesos de inclusión social y cultural, entre otros temas. Aunque la discusión hasta ahora se ha hecho sobre todo en los países receptores del centro del sistema-mundo, ya empezó a extenderse a los países del sur (GMG, 2013). América Latina no ha estado exenta de estas tendencias, aumentando los desplazamientos dentro de la región de manera importante en los últimos años (Martínez, 2011), generándose múltiples desafíos para la convivencia multicultural y el desarrollo social, y convocando el interés científico desde las ciencias sociales.

Este flujo migratorio Sur-Sur se debe tanto a factores externos como internos. La reciente crisis económica internacional (iniciada en 20072008 y cuyas consecuencias perduran hasta hoy), tiene como trasfondo la reforma estructural neoliberal que profundiza la explotación capitalista en un contexto histórico poscolonial y de división internacional y sexual del trabajo (Wallerstein, 2007; Falquet, 2009). Estos fenómenos macro, producto de un modelo de desarrollo extractivista orientado a las exportaciones, han aumentado las asimetrías entre los países y las desigualdades en cada sociedad, tendiendo a crecer la exclusión social y la pobreza, lo que ha desembocado en grandes movimientos hacia Estados-nación que brinden mayor seguridad (Solimano, 2008).

Históricamente, y hasta la actualidad, en Sudamérica Colombia ha sido el país que ha generado mayores flujos de emigración (Martínez y Orrego, 
2016). Los principales Estados de acogida de población colombiana han sido Estados Unidos, España, Venezuela y Ecuador, pero en la última década los flujos migratorios colombianos se han incrementado hacia destinos que antes no eran frecuentes, entre ellos Chile (Mejía, 2012). ${ }^{5}$ Importantes retos económicos y políticos ha debido enfrentar Colombia en las últimas tres décadas debido al colapso en los precios internacionales del café, la crisis petrolera y el auge del conflicto armado, en el contexto de la expansión del tráfico de drogas hace ya seis décadas, forzando a muchas personas a desplazarse internamente y/o abandonar su país natal, habiendo hoy un total de 4700000 colombianos/as residiendo en el exterior (Cárdenas y Mejía, 2006). Ahora bien, no sólo los grupos más vulnerables se movilizan desde Colombia hacia otros países del sur, sino que una parte de estas migraciones son protagonizadas por sujetos de estratos medios y altos, algunos con estudios técnicos o universitarios. Así, la diversidad de clase y "raza" distingue los recientes flujos Sur-Sur, entre ellos el colombiano, lo que también incide en sus patrones de integración socioeconómica y sociocultural.

Por su parte, desde hace algunos años Chile ha pasado a ser uno de los países de la región que son elegidos como destino de los migrantes SurSur. Según los datos del DEM (2016), los residentes extranjeros en el país pasaron de 212935 en 2005, 1.3 por ciento de la población de ese año, a 410988 inmigrantes en el año 2014, representando 2.3 por ciento de la población nacional, es decir, la población inmigrante ha aumentado en 93 por ciento en los nueve años que abarca dicho estudio. ${ }^{6}$ En este contexto, el colombiano ha pasado a ser el cuarto colectivo de inmigrantes más importante en el país. Según el DEM (2016), hasta el año 2014 solamente los inmigrantes provenientes de los tres países limítrofes - peruanos (130 329), argentinos (66 899) y bolivianos (36 036) — superaban en número a los colombianos, los cuales ascendían a 25038 personas, 6.1 por ciento de los residentes extranjeros del país. Sin embargo, el colectivo colombiano presenta un aumento muy superior al de los otros tres países mencionados, de 394 por ciento entre 2005, cuando sólo había 5066 residentes en Chile,

\footnotetext{
${ }^{5}$ La diversificación de los destinos de la emigración colombiana van más allá de Chile. En Argentina, por ejemplo, en los últimos años se ha visto un incremento similar al que se ha producido en Chile (OIM, 2016).

${ }^{6}$ El fenómeno de la inmigración en Chile resalta la importancia de contar con información estadística rigurosa. Lamentablemente en estos momentos Chile no cuenta con datos estadísticos oficiales sobre la población inmigrante ni sobre el saldo migratorio (inmigrantes menos emigrantes), ya que a la falla del Censo 2012 se le suma la carencia de registros administrativos y de encuestas especificas sobre el tema. Se deberá esperar los resultados del Censo 2017 para contar con información estadística oficial sobre inmigración, pero una más importante producción de información estadística continua a través de registros no se avizora en el corto ni mediano plazo.
} 
y 2014, mientras peruanos, argentinos y bolivianos crecieron, respectivamente, 198, 25 y 188 por ciento en igual periodo (DEM, 2016). Así, la importancia de esta comunidad no sólo proviene de su número, sino especialmente de su tendencia de crecimiento. En términos de su distribución territorial, los inmigrantes colombianos/as se concentran principalmente en la Región Metropolitana, 61 por ciento, y en la Región de Antofagasta, 12.4 por ciento (DEM, 2016). ${ }^{7}$

A mediano plazo los flujos migratorios pueden cambiar de manera impredecible. Factores como el proceso de paz entre el Estado colombiano y la guerrilla de las FARC, pueden hacer que disminuya el flujo migratorio de colombianos/as hacia Chile, particularmente de refugiados. Lo contrario puede pasar si continúa la crisis económica y política en Venezuela, uno de los países que ha sido históricamente destino preferente de la emigración colombiana, lo que puede hacer que los emigrantes colombianos opten por otros destinos, entre ellos Chile. Sin embargo, si bien a mediano plazo la tendencia migratoria puede variar de manera incierta, es altamente probable que en el corto plazo este flujo continúe su aumento. Esto porque el incremento en el flujo migratorio provoca un fortalecimiento en las redes transnacionales, lo que conlleva un aumento en la capacidad de los inmigrantes para convocar familiares y conocidos. Además, se ha mantenido la diferencia entre Colombia y Chile en indicadores económicos como el ingreso per cápita, lo que puede actuar como elemento catalizador de la migración. Estos factores hacen que a corto plazo sea previsible que se mantenga el incremento de este flujo migratorio, lo que hace necesario estudiar este colectivo de inmigrantes y sus procesos de integración y exclusión.

\section{MARCo TEÓRICo}

\section{La geocultura del sistema-mundo}

Tal como lo enfatizaron los autores de la Escuela de Frankfort (por ejemplo Adorno, 2001), es de central importancia establecer el marco de la totalidad que tiene como referente un estudio social, por más empírica que sea su orientación. Para el estudio de la migración y sus procesos de integración y exclusión en determinada sociedad, no parece adecuada la referencia de totalidad tradicional centrada en "la sociedad" —el "nacionalismo metodológico" - sino que estimamos debe reorientarse al ámbito del sistema-mundo capitalista. Esto no sólo porque la migración internacional

\footnotetext{
${ }^{7}$ Sobre afro-colombianos en la Región de Antofagasta, ver Echeverri (2016).
} 
es un fenómeno que necesariamente traspasa las fronteras de cualquier sociedad, también porque los fenómenos que ocurren tanto en la sociedad de expulsión como en la de recepción expresan su inserción económica, política y cultural en el sistema-mundo. Tal como lo plantearon Cardoso y Faletto (1979) al estudiar las clases sociales en la situación dependiente de los países latinoamericanos, las características de la estructura socioeconómica "interna" son una expresión de factores "externos" — la forma de la inserción periférica de las sociedades latinoamericanas en el sistema capitalista-, siendo finalmente incorrecto separar los factores "externos" de los "internos" en el análisis de las características socioeconómicas de un país. El mismo planteamiento se puede hacer extensivo a los fenómenos socioculturales como el racismo, que afecta directamente la dinámica de integración/exclusión de las distintas comunidades de inmigrantes, los cuales pueden comprenderse de mejor manera al considerar que los hechos "internos" son expresión de factores "externos", en este caso, la geocultura del sistema-mundo.

En el planteamiento central de la Perspectiva del Sistema-Mundo se pone especial énfasis en la dimensión económica, específicamente en la división internacional del trabajo, para definir el carácter sistémico de la economía-mundo capitalista. Según Wallerstein (2003), en el marco del sistema-mundo se produce una división del trabajo que traspasa las fronteras estatales, abarcando el conjunto de su geografía intercontinental, lo que hace que las partes del sistema, desde su origen en el siglo XV, no sólo mantengan un vínculo económico con el resto del sistema, sino que estructuran sus economías en función de su posición - céntrica, semiperiférica o periférica- en el sistema. Tomando estos planteamientos como base, desde la Perspectiva Decolonial se enfatiza que el sistema-mundo "moderno/colonial" también posee una geocultura que surge con la conquista de América (por ejemplo, Castro-Gómez, 2000; Lander, 2000 y Mignolo, 2000). A partir de la conquista de América se habría generado una interpretación cultural del papel de Europa y de los demás pueblos que eran parte del sistema mundial. El papel dominante de Europa y su sometimiento de los demás pueblos se interpreta en base a concepciones culturalistas y racistas, con lo que se conforma una cultura del conjunto del sistemamundo, a partir de la cual se organizan las relaciones intersubjetivas entre dominantes y dominados, correspondiendo el dominio militar y económico de Europa con su dominio cultural.

Hacia los siglos XVII y XVIII, y particularmente con la Ilustración, esta interpretación cultural se plasma en un discurso eurocéntrico sobre 
la modernidad que plantea a Europa como la culminación del desarrollo sociocultural de la humanidad, posicionándose a los demás pueblos, culturas y "razas" como fases evolutivas previas a Europa y por lo tanto como inferiores a ella (Castro-Gómez, 2000; Lander, 2000 y Mignolo, 2000). En este contexto se producen las independencias políticas de los países latinoamericanos. Con ellas el paradigma eurocéntrico de interpretación cultural no cambia, ya que los procesos independentistas fueron liderados por las minorías blancas que dominaban la vida social, económica y cultural de las colonias. Las clases que mantuvieron su dominio en Latinoamérica se identificaban culturalmente con Europa, y adoptaron como propia la perspectiva eurocéntrica, imponiendo esta visión cultural en América Latina, con lo que se re-articuló y mantuvo la colonialidad cultural en la nueva situación sociopolítica y geopolítica. Así, con el proceso de independencia no se produjo una descolonización cultural, el final del colonialismo no significó el final de la colonialidad (Restrepo y Rojas, 2010).

En este contexto, la "raza" es empleada como un patrón de clasificación social, de jerarquización, que se fundamenta en una distinción en base a fenotipos y que se justifican en términos biológicos (Restrepo y Rojas, 2010). Esta distinción es empleada en la división del trabajo que se impuso en las colonias americanas al ser incorporadas al sistema-mundo capitalista, definiéndose el trabajo esclavo para los "negros" africanos, el trabajo servil obligatorio para los "indios" americanos, y el trabajo remunerado para los "blancos" europeos, por lo que la "raza" se emplea como un instrumento de dominación social y explotación económica. La división racial del trabajo se articula con la producción de una intersubjetividad mundial, de unas subjetividades "geoculturales" interrelacionadas - indios, negros, blancos, entre otros-, que define las relaciones entre Europa y los europeos con el resto del mundo y sus habitantes en términos jerárquicos. La culminación del uso de la idea de raza para la clasificación jerárquica de las poblaciones se alcanza en el siglo XIX y en la primera mitad del siglo $\mathrm{XX}$, cuando el racismo se conforma como un complejo de ideas y teorías en torno al principio de la jerarquía racial, como el darwinismo social. Es central señalar que la idea de raza no sólo produce subjetividades entre los colonizadores dominantes, también coloniza el imaginario de los colonizados, que comienzan a mirarse desde la visión del dominador racista. Así, desde la conquista de América y el subsiguiente establecimiento de la división racial del trabajo, han perdurado las ideas asociadas de una jerarquización biológico-cultural que se emplean para justificar y naturalizar las relaciones de dominación (Restrepo y Rojas, 2010). 


\section{Sociedades multiculturales: el extranjero/inmigrante, entre lo próximo y lo distante}

Los flujos migratorios tienen como consecuencia que los Estado-nación de recepción devengan en colectivos étnicamente múltiples y/o aumenten su pluralidad cultural. Para caracterizar, en términos generales, a las sociedades de recepción, resultan importantes los planteamientos de Kymlicka (1996), quien presentó una propuesta para los desafíos de las sociedades multiculturales. Este autor realiza distingue entre Estados multinacionales y Estados poli-étnicos. Los primeros son aquellos en los que han sido incorporadas una o más culturas minoritarias concentradas territorialmente y que fueron previamente autogobernadas, mientras que los Estados poliétnicos son producto de la inmigración. En Chile se presentan rasgos de ambas características. Por una parte, hay culturas minoritarias con algún grado de concentración territorial, que fueron incorporadas al Estado-nación chileno, antes o después de su independencia. Esta incorporación fue realizada con diferentes grados de violencia buscando, hasta hace pocos años, su "chilenización". Por otra parte, en diferentes momentos históricos se han incorporado al Estado-nación chileno diversas corrientes migratorias, particularmente desde Europa y los países limítrofes, sin conformar grupos étnicos diferenciados. Así, la "chilenización" de los pueblos originarios y la incorporación de diferentes corrientes migratorias, han tenido como resultado una población fundamentalmente "mestiza". Sin embargo, este mestizaje es heterogéneo, con mayores o menores componentes de población "europea" u "originaria" dependiendo de los estratos socioeconómicos de la población, con rasgos fenotípicos más "europeos" en los estratos altos, y rasgos más "originarios" en los estratos bajos. En el marco racista de la geocultura del sistema-mundo capitalista, esto ha dado como resultado un entrecruzamiento del racismo y el clasismo, una cultura donde se valorizan los rasgos fenotípicos europeos y se los vincula al éxito económico, y se desvalorizan los rasgos fenotípicos característicos de los pueblos originarios y se los vincula a la pobreza. En este contexto general se insertan los colectivos de inmigrantes en la actual sociedad chilena.

Por otra parte, las características étnicas y raciales de los inmigrantes pueden tener diferentes relaciones con la sociedad de recepción. Guibernau (2009) distingue tres tipos de situaciones: i) los inmigrantes que se parecen socio-culturalmente a la mayoría de la población receptora; ii) los que al principio fueron discriminados y que tienden a formar comunidades étnicas, pero que se han integrado del todo a la sociedad de acogida y han adquirido la ciudadanía; iii) finalmente, los inmigrantes con rasgos 
fenotípicos que les distinguen de la mayoría de la población, viviendo en comunidades étnicas relativamente cerradas y que suelen ser objeto de discriminación racial y marginación socioeconómica. Los diferentes tipos/ perfiles de inmigrantes colombianos y colombianas en Chile podrían, dada su pluralidad, responder a los tres tipos descritos por Guibernau (2009). Su forma de inserción en una sociedad como la chilena, con rasgos, siguiendo a Kymlicka (1996), multinacionales y poli-étnicos, estará mediada por la forma que adquiere la geocultura del sistema-mundo capitalista en Chile, y cómo desde ella se establecen relaciones intersubjetivas con los diferentes colectivos de inmigrantes.

\section{Análisis}

\section{Caracterización general de los inmigrantes colombianos/as en Chile}

Para la caracterización general del colectivo de inmigrantes colombianos se utilizan las bases de datos que dispone el DEM, las cuales entregan información sobre diferentes variables como sexo, edad, nivel educacional, actividad, profesión, comuna de residencia al momento de solicitar los documentos, tipo de visa entregada, año de entrega de las visas y de los permisos de residencia definitiva, entre otros. Buscaremos caracterizar la dinámica migratoria reciente y aproximarnos a las características socioeconómicas, a los estratos sociales, de los inmigrantes.

Como se señaló, según el DEM (2016), en el año 2014 habían 25038 colombianos y colombianas residiendo en el país, constituyéndose en el cuarto colectivo de inmigrantes más numeroso luego de los que conforman los ciudadanos de Argentina, Bolivia y Perú. Junto al stock de migrantes, también es importante tener una idea de la evolución del flujo migratorio, para acercarse así a la dinámica migratoria. Para ello empleamos los datos que se disponen sobre la entrega de permisos de residencia definitivas y de visas temporales en el DEM. ${ }^{8}$

$\mathrm{Al}$ observar la tendencia que muestran en los últimos años tanto el otorgamiento de permisos de residencia definitivos como el de visas tempora-

\footnotetext{
${ }^{8}$ Cabe señalar que las estadísticas disponibles hacen referencia a los documentos entregados, no a personas, es decir, las cifras indican la cantidad de documentos entregados al año y no la cantidad de personas que los recibieron. Esto quiere decir que una misma persona a lo largo del tiempo puede recibir más de un documento, en el caso de las visas temporales incluso más de un documento en un mismo año y múltiples visas a lo largo de un período de varios años. En el caso de los permisos de residencia definitiva también pueden entregarse más de un documento a una misma persona, aunque esto es relativamente excepcional, y la entrega tiene que ser en diferentes años.
} 
les (Tabla 1), ${ }^{9}$ se destaca que hay un sostenido aumento en ambos indicadores. En el período que transcurre entre los años 2005 y 2015 se pasa de 403 permisos de residencia definitiva y 1737 visas temporarias otorgadas en el año 2005 a 9093 permisos de residencia definitiva y 35445 visas temporarias en 2015. Una situación similar se produce en la Región Metropolitana, donde se pasa de 302 permisos de residencia definitiva y 1216 visas temporarias en 2005 a 4588 permisos de residencia definitiva y a 19 032 visas temporarias en el año 2015.

En la Tabla 2 se puede ver el porcentaje de crecimiento interanual de los permisos de residencias definitivas y las visas temporarias para Chile y la Región Metropolitana. Se puede apreciar que año con año se ha venido produciendo un aumento en el otorgamiento de ambos documentos. La única excepción es el período entre los años 2009 y 2010 para los permisos de residencias definitivas, donde se produce una disminución en la entrega de estos documentos tanto a nivel del país como de la región. ${ }^{10}$

El promedio de los crecimientos porcentuales interanuales que muestra la Tabla 2 es de 42.5 por ciento en el caso de los permisos de residencia definitivas para el país, de 36.4 por ciento para las visas temporarias a nivel país, y de 36 y 32.7 por ciento respectivamente para la Región Metropolitana. Sin embargo, si se divide el período en dos quinquenios, se puede apreciar (Tabla 3) que el crecimiento de la dinámica migratoria va en aumento.

La Tabla 3 muestra que en todos los casos el segundo quinquenio tiene un mayor porcentaje de crecimiento que el primero, siendo la mayor diferencia la de los permisos de residencia definitiva a nivel del país, que pasaron de 260.5 por ciento de aumento en el primer quinquenio a 525.8 por ciento en el segundo. Así, el flujo migratorio no sólo se estaría incrementando en términos absolutos, también hay un aumento en términos relativos, pues está aumentando el ritmo de crecimiento del flujo migratorio. Para finalizar esta sección, cabe destacar que el porcentaje de incremento anual en el periodo de estudio supera con largueza mil por ciento en cualquiera de los cuatro niveles analizados, superando dos mil por ciento para el otorgamiento de permisos de residencia definitiva a nivel país.

\footnotetext{
${ }^{9}$ Todas las tablas han sido elaboradas a partir del procesamiento propio de las bases de datos publicadas por el Departamento de Extranjería y Migración del Ministerio del Interior y Seguridad Pública en http://www.extranjeria.gob.cl/estadisticas-migratorias/

${ }^{10}$ Esta disminución probablemente se relaciona con el gran aumento de permisos de residencia definitivas que se produce en el año 2009 , que tiene más de 100 por ciento de incremento con respecto al año 2008 para el país, por lejos el incremento interanual más significativo del periodo. Al respecto Rojas y Silva (2016: 17) señalan: "Es necesario mencionar que el peak que se visualiza el año 2009 en el otorgamiento de permanencias definitivas a diversos colectivos, se explica por el proceso de regularización masiva que se efectuó el año 2007, pues las personas que obtuvieron una visa ese año pudieron obtener un permiso de permanencia definitiva en el año 2009".
} 
Integración y exclusión de inmigrantes colombianos recientes en Santiago de Chile ... /E.N. GISSI y G. GHIO

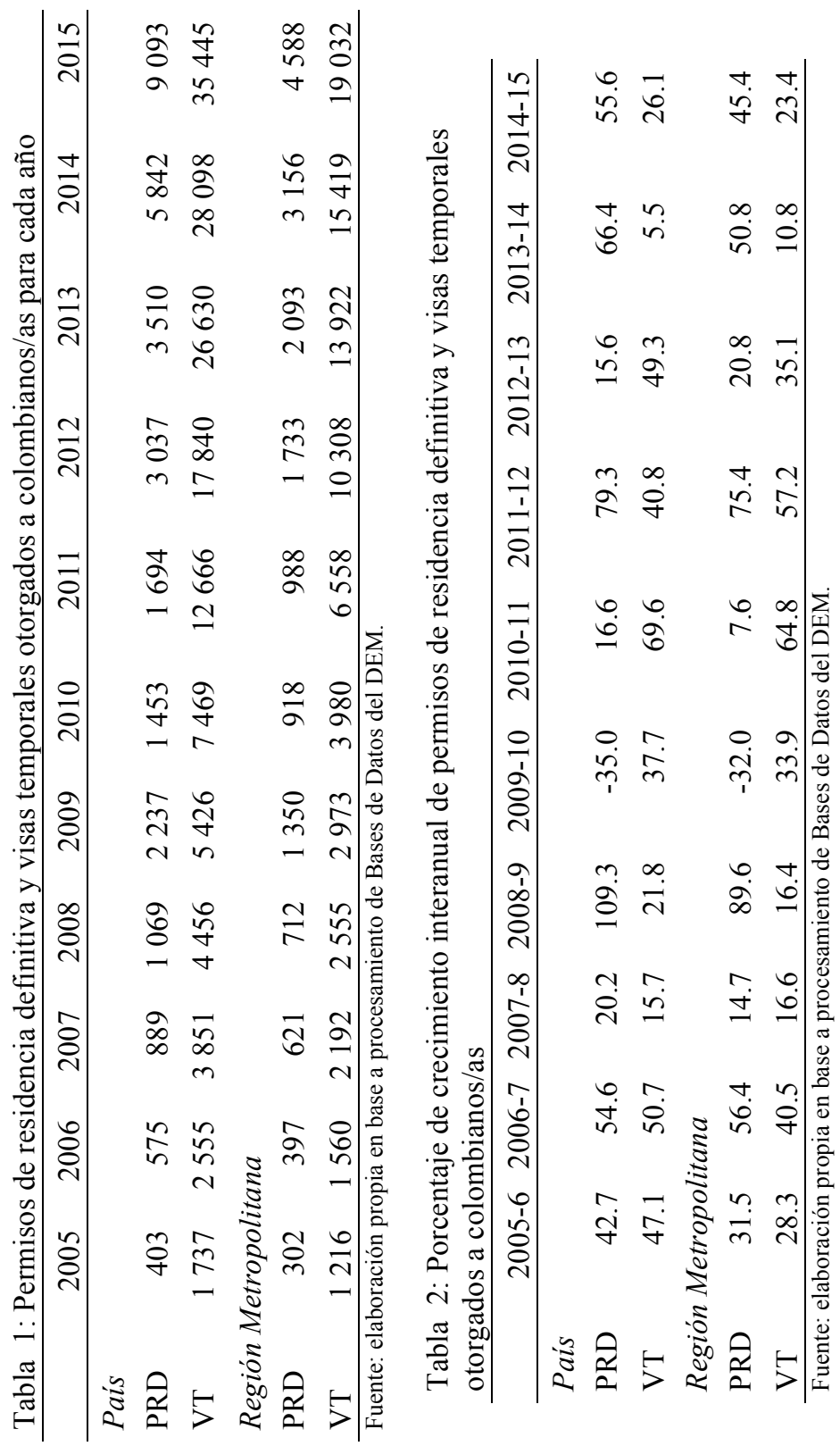


Tabla 3: Porcentaje de crecimiento por quinquenios de permisos de residencia definitiva y visas temporales otorgados a colombianos/as

\begin{tabular}{lrrrrrr}
\hline & \multicolumn{3}{c}{ País } & \multicolumn{3}{c}{ Región Metropolitana } \\
\cline { 2 - 7 } & $2005-10$ & $2010-15$ & $2005-2015$ & $2005-10$ & $2010-15$ & $2005-2015$ \\
\hline PRD & 260.5 & 525.8 & 2156.3 & 204.0 & 399.8 & 1419.2 \\
VT & 330.0 & 374.6 & 1940.6 & 227.3 & 378.2 & 1465.1 \\
\hline
\end{tabular}

Fuente: elaboración propia en base a procesamiento de Bases de Datos del DEM.

En cuanto a la heterogeneidad socioeconómica de la comunidad colombiana, a través de la variable "Profesión" de la base de datos de Permisos de Residencia Definitivas que otorga el DEM, nos hemos podido aproximar a la estratificación socioeconómica de este colectivo de inmigrantes. Las ocupaciones son una de las variables proxy más utilizadas para producir una estratificación socioeconómica de una población, y la variable "profesión" de la base de datos del DEM se puede usar para aproximarse a la ocupación de los sujetos. La información de esta variable debe ser tomada con cautela por, entre otros motivos, ser obtenida a partir de la auto-declaración de los inmigrantes sin una posterior corroboración, por contener en muchos casos "profesiones" poco claras (por ejemplo "Ayudante"), y porque no necesariamente la profesión declarada efectivamente puede ser ejercida en el desempeño laboral habitual del inmigrante. Sin embargo, considerando estas precauciones, estimamos que la información puede ser utilizada para realizar una caracterización socioeconómica general del colectivo de inmigrantes colombianos.

Para tener mayores precauciones, del total de 29802 Permisos de Residencia Definitivas a considerar se eliminaron 419 casos de profesiones con frecuencias iguales o menores a cinco y se eliminaron 5984 casos de profesiones que consideramos "indeterminadas", que no se podían asignar a un estrato social ya que se requeriría mayor información (por ejemplo 3384 "empleados", 435 "ayudantes" y 305 "administradores"). Además en la base habían 5562 estudiantes y 2737 personas inactivas (dueñas de casa, jubilados/as y oficios religiosos) que tampoco se pueden asignar a un estrato socioeconómico sin tener referencias del resto de su hogar, lo cual no existe. De esta manera, se eliminaron 14702 casos de los 29802 originales.

Los restantes 15100 casos consideramos que podían ser distribuidos en grupos socioeconómicos, tomando la nueva precaución de utilizar grupos muy amplios, por lo que sólo los distribuimos entre estratos "alto", "medio" y "bajo". Para el estrato alto consideramos profesiones ligadas 
al ámbito de la medicina (médicos y odontólogos), a las ingenierías y las finanzas, lo que dio un total de 3474 casos.

En el estrato medio asignamos a una amplia gama de profesionales y técnicos, como enfermeros, profesores, electricistas, psicólogos o abogados. Además consideramos oficios que no necesariamente requieren cualificaciones formales, pero que requieren ciertos grados de especialización y cualificación que hacen que sus remuneraciones sean relativamente elevadas, como modelos, mueblistas o pasteleros. También incorporamos algunas categorías que, aunque son profesiones indeterminadas, estimamos que probablemente pertenecerían a un estrato medio, como las categorías de "técnico", "cientista" o "investigador". De esta manera totalizamos 4 510 casos.

Para el estrato bajo consideramos oficios de baja cualificación y valoración en el mercado laboral, como garzones, conserjes, cocineros, obreros, asesoras del hogar, entre otros. El total de casos que quedaron asignados al estrato socioeconómico bajo fue de 7116.

Si bien la asignación de "profesiones" a los tres estratos socioeconómicos es compleja, y es debatible la asignación de algunas de ellas a un estrato u otro, estimamos que el resultado final de esta aproximación corrobora la percepción de que la comunidad de inmigrantes colombianos/ as efectivamente tiene una variabilidad socioeconómica considerable, distribuyéndose contingentes importantes entre los estratos socioeconómicos alto, medio y bajo. Así, esta aproximación entrega una cifra de 23 por ciento casos en el estrato alto, 29.9 por ciento en el estrato medio, y 47.1 por ciento en el estrato bajo.

Esta variabilidad socioeconómica se refleja en las entrevistas analizadas, ya que entre los interlocutores se pueden distinguir personas de diferentes profesiones, actividades laborales, niveles educacionales y que habitan espacios urbanos muy disímiles - como La Dehesa en la comuna de Lo Barnechea, zona residencial de muy altos ingresos, o la población Pablo de Rokha en la comuna de La Pintana, una de las más pobres del Gran Santiago-.

\section{La integración/exclusión de los inmigrantes colombianos/as}

A continuación se analizarán las características que adquiere la integración/ exclusión de los inmigrantes colombianos/as en la Región Metropolitana, los modos como se insertan en diversos ámbitos sociales, así como las dificultades que enfrentan, entre otros motivos, por los prejuicios vinculados a este colectivo, como la asociación de Colombia con el narcotráfico. En 
el análisis se distinguirán las diferencias que se producen entre los inmigrantes de distintos estratos socioeconómicos y "razas", buscando conocer si cambian las formas de integración de los distintos grupos. El estudio se fundamenta en más de cincuenta entrevistas en profundidad y cuatro historias de vida realizadas entre el año 2014 y el año 2016. El análisis se realiza desde la propia vivencia de los inmigrantes entrevistados/as en torno a las dimensiones vinculadas a su integración/exclusión.

El discurso dominante de los entrevistados apunta a que tienen una buena integración a la sociedad chilena. Prácticamente todos los entrevistados relatan experiencias positivas en Chile, su aprecio del país y de sus habitantes. En general el discurso hace referencia a una integración armónica, en las que predominan buenas experiencias, como lo señala Claudia (mestiza/“blanca”, medio-alto $)^{11}$ : (¿Ha sido fácil integrarse a Chile?) Sí, ha sido bonito. Y he conocido gente agradable, bonita, de buen trato ehh... amable... bien". ${ }^{12}$ Acentuando esta visión, algunos entrevistados señalan que esta buena experiencia general logró quebrar las predisposiciones negativas iniciales que tenían, ya que al tener un proyecto de retorno definido no querían establecer mayores relaciones en Chile. Este fue el caso de Diana B. (mestiza/“blanca”, alto):

"Yo estaba súper cerrada, yo tenía, no sé, cómo una barrera que yo misma me puse para no vincularme acá, porque yo... mi plan era regresar. (Sin embargo) tuve muy buenas amigas en la universidad, muy buenas, nada que decir...la verdad es que mi grupo es chileno, o sea mi gente es más chilena que colombiana".

Esta visión general lleva a varios entrevistados a afirmar que a los chilenos les agradan los colombianos, que les gusta su personalidad, alegría, forma de ser, incluso de hablar. En esta línea Francisco (mestizo/"blanco", bajo) señala:

“...hasta ahora bien, todos me han atendido bien, en el restaurante donde trabajé igual, aquí en la fábrica, en la calle, toda la gente los chilenos han sido bien conmigo, y con muchos colombianos también, me han dicho que el chileno no es que sea como muy indiferente con el colombiano, de hecho la mayoría de chilenos y chilenas les gusta mucho el colombiano, porque les agrada".

\footnotetext{
${ }^{11}$ Para cada cita a un entrevistado/a se especificará si es afrocolombiano o mestizo/“blanco" y su estrato socioeconómico: alto, medio-alto, medio, medio-bajo o bajo.

${ }^{12}$ En las citas de las entrevistas se respeta la forma como se expresan los entrevistados/as, por lo que pueden encontrarse problemas gramaticales en estas referencias además de dichos y expresiones propias de Chile y Colombia.
} 
Esta buena experiencia de integración que se manifiesta en el discurso dominante, no soslaya un proceso de adaptación a las diferencias culturales que sienten con los/as chilenos/as, por tanto a esfuerzos que se deben hacer para adaptarse y comprender los códigos culturales de la sociedad de recepción. Una característica cultural a la que se alude reiteradamente, y que inicialmente cuesta asimilar, sería una cierta "frialdad" o "distancia" que tendrían los chilenos en su forma de relacionarse, no con los inmigrantes en particular sino de forma general. Por ejemplo Aristarco (mestizo/"blanco", medio-alto) señala:

“Cómo te han tratado los chilenos? En general bien, no tengo ninguna queja la verdad. En un principio es fuerte el impacto porque son mucho más fríos a la hora de hacer una relación, y son mucho más herméticos a la hora de que alguien nuevo entre a un grupo de amigos".

La situación positiva general a la que hace referencia el discurso dominante de los inmigrantes debe ser matizada por dos factores. Por una parte, prácticamente todos los entrevistados, incluso los que se sienten más a gusto en Chile, pueden relatar alguna experiencia de discriminación que les ha tocado vivir. Nos referimos aquí a formas de discriminación que, por una parte, son de baja intensidad y, por otra, son relativamente excepcionales, situaciones que, como veremos más adelante, son diferentes en el caso de los afrocolombianos de los estratos socioeconómicos más bajos. En el discurso dominante del colectivo de inmigrantes colombiano, se plantea que las discriminaciones que han vivido no afectan de manera importante el proceso de integración.

Por otra parte, el discurso dominante también hace referencia a situaciones complejas en un espacio específico donde se enfatiza que se reciben malos tratos, discriminación y arbitrariedades, este espacio es el de las instituciones relacionadas con los trámites de inmigración, ya sea en la frontera como en las oficinas de la Policía de Investigaciones (PDI), el Registro Civil y el DEM.

Junto a este discurso dominante, surge el relato de quienes sí señalan haber sufrido discriminación, discurso que se relaciona directamente con el problema del racismo, el cual, a su vez, se entrecruza con la estratificación socioeconómica, no sólo porque la mayor parte de los inmigrantes afrocolombianos entrevistados pertenecen a estratos bajos y medios-bajos, sino también porque, como lo mostraremos a continuación, los actos de discriminación y racismo son más frecuentes en los entornos en los que se desenvuelven los inmigrantes de los estratos socioeconómicos más bajos. 
En las entrevistas se puede apreciar que los inmigrantes que se insertan en sectores socioeconómicos medio-altos o altos tienden a tener una buena inserción en el medio social chileno, en general poseen amigos chilenos, y en varios casos tienen parejas chilenas o hacen referencia a amigos o conocidos que las tienen. Prácticamente no hay afrodescendientes entre estos inmigrantes de estratos medios-altos. Por su parte, en los estratos mediosbajos y bajos se aprecian mayores problemas de integración, mayor dependencia de la comunidad de colombianos o de inmigrantes en general y, entre los problemas de integración destacan la discriminación que afecta a los inmigrantes afrocolombianos, que mayoritariamente se insertan en estos estratos. Nos detendremos primero en el aspecto socioeconómico de la discriminación para luego centrarnos en el racial.

Los inmigrantes de estratos bajos y medios-bajos enfrentan mayores niveles de discriminación y dificultades de inserción y adaptación que los inmigrantes de estratos altos y medios-altos. Algunos de los inmigrantes de estratos bajos y medios-bajos no afrodescendientes dan cuenta de experiencias discriminatorias y problemas que les ha tocado vivenciar que, si bien no son de alta intensidad, como luego se verá con los afrocolombianos, se experimentan de manera relativamente frecuente, cotidiana, por lo que pueden constituir un problema para su integración. Lucy (mestiza/“blanca", bajo) señala: "...la verdad es que he encontrado de todo, porque he encontrado gente muy amable, gente muy linda, muy linda, pero también gente que, no sé, te discrimina mucho", y en la misma línea Francisco (mestizo/“blanco”, bajo) señala: "sí, algunas veces sí (he sido discriminado) pero bueno... no le voy a prestar atención a amargarme, no tengo tiempo".

Además, los inmigrantes de estratos bajos y medios-bajos también están expuestos a situaciones en las que es más probable que reciban algún maltrato, al igual que la población chilena de esos estratos, pero en el caso de ellos las ofensas se expresan en términos discriminatorios y xenofóbicos. Por ejemplo, en los consultorios de salud pública los pacientes pueden recibir agravios que, muy probablemente, no se producen en los centros de salud privada. Esto lo señala Vanessa (mestiza/“blanca”, medio-bajo): "en un consultorio ... las matronas... fueron así como pesadas conmigo, ahh, me dijo 'estas extranjeras vienen y creen que una las tiene que atender al tiro' una cosa así”. Así mismo, al postular a trabajos también se ven expuestos a situaciones de maltrato que probablemente no vivencian quienes postulan a trabajos propios de los estratos altos y medios-altos. Por ejemplo, Andrés (mestizo/“blanco", medio-bajo), al buscar trabajo como 
mesero vivió ese tipo de situaciones: "Si obvio, pues claro cómo llega uno, como que 'no, no puedes trabajar aquí porque no recibimos extranjeros', ya bueno chao. Es una forma de discriminación (me pasó) buscando en bares, en Bellavista... Si en varias partes: "no, extranjeros no"'.

Esto contrasta con las experiencias de los inmigrantes de estratos altos y medios-altos. Como ya se señaló, varios no consideran haber sufrido ningún tipo de discriminación, y quienes sí las han vivido, las consideran excepcionales, acontecimientos aislados, que no afectan su vida cotidiana. Por ejemplo, Alejandro (mestizo/“blanco", medio-alto) señala: "(¿Se ha sentido discriminado?) Sí, un par de ocasiones sí...casos aislados, que es normal ... yo entiendo eso, pero no me he sentido así en términos generales discriminado".

Esta diferencia en el trato según los estratos socioeconómicos es reconocida y explicitada por los propios inmigrantes. Diana (mestiza/“blanca", medio) lo señala:

"Hay una cosa acerca de la xenofobia que se está manifestando cada vez más fuerte ... como trabajé en Providencia y vivo como con gente que estudia letras, son periodistas, gente que igual fue a la universidad.... yo no he tenido que vivir eso. Pero los domingos me gusta ir a ferias por ejemplo, y ahí es donde encuentras la real xenofobia, curiosamente son las clases bajas, las que tienen alergia al extranjero, pero las clases altas o medias e intelectuales son maravillosos ... hay una xenofobia que crece cada vez más, pero es entre la gente pobre, lo que es lo triste... sí, y se compiten los empleos ¿viste?, como se compiten los empleos con los colombianos, con los peruanos, y ahí es donde está la real xenofobia, que es triste, porque los pobres son pobres en todos lados... y en vez de estar pendientes del real opresor, están pendientes del colombiano, del peruano..."

Teniendo esta diferencia entre los estratos en consideración, al introducir el problema del racismo, se puede comprobar que los afrocolombianos sufren una discriminación muy superior a sus compatriotas del mismo estrato social. Prácticamente todos los entrevistados afrocolombianos relatan haber sido víctimas de discriminación racial en múltiples ocasiones. Hostigamiento en la calle, no ser atendidos adecuadamente en los lugares de compras o trámites, buses del transporte público o taxis que no se detienen cuando ellos lo solicitan, tratos arbitrarios en las fronteras o en los trámites de visados, personas que se niegan a ser atendidos por ellos, insultos e incluso agresiones físicas, todas estas situaciones son relatadas por inmigrantes afrodescendientes. Así lo señala Marta (afrocolombiana, medio-bajo): “Aquí hay mucha discriminación... ¡demasiado! Que porque 
uno es colombiana, que porque uno es negra". Y plantea directamente el problema del color de piel como causa de la discriminación: “....aquí hay mucha gente que no lo quiere a uno por lo negro". Esto también lo afirma Diego H. (afrocolombiano, bajo):

“...te tratan con palabras racistas y todo eso, eso da mucha rabia... te dicen negro cuando ni siquiera son blancos, ¿ya me entiende? [...] yo creo que es una falta de cultura, eso sí me molesta, negro yo-no-sé-qué cuando ni siquiera son blancos $[\ldots]$ o sea no sé por qué te discriminan, te tratan como de discriminar por el color...".

Los actos de exclusión racistas a los que hacen referencia los entrevistados afrocolombianos claramente son de una intensidad significativamente superior a las discriminaciones que enfrentan los no afrodescendientes. Como puede verse en las siguientes citas, estos actos de discriminación pueden tener cargas de violencia importantes, incluso físicas: Marta (afrocolombiana, medio-bajo): “(¿La han tratado mal?) Sí. (¿Cómo qué por ejemplo?) Que negra colombiana, que falopera, que no sé qué... que conchetumadre, esas. (¿Y de la nada?) ¡De la nada!”; Diego H. (afrocolombiano, bajo):

“...una vez que estaba en un videojuego...cuando llego uno y empezó a decirme 'que se puso oscura la cosa, que no sé qué, que está negra la cosa, que negro yo no sé qué'...y que una cosa y que otra, y pues, que él le había pegado a todos ahí en la población, y pues, empezó a gritarme, a sonarme los dedos aquí... y yo callado, hasta que me azaré y le pegué un cabezazo y le partí la nariz, y ya... Y ya y después dijeron... que me iban a sacar de la casa a balas, que no sé qué, y una cosa, y la otra... al principio yo me quedé como asustado porque en Colombia cuando te dicen que te van a sacar de la casa a balas es porque es verdad...".

Los espacios públicos son lugares particularmente delicados en relación a la discriminación racial, ya que el racismo y la xenofobia se pueden manifestar con particular dureza por la sensación de impunidad que puede tener quien discrimina, al verse escudado por el anonimato y por la masa de sus compatriotas, y porque es más probable que una reacción de quien es discriminado pueda derivar en actos de violencia.

En estos espacios son manifiestas las diferencias en las experiencias entre los afrocolombianos y los inmigrantes no afrodescendientes, probablemente porque el fenotipo del colombiano "blanco" y mestizo no es muy diferente al chileno, por lo que su condición de extranjero puede pasar desapercibida. Por ejemplo, Claudia (mestiza/“blanca”, medio-alto) señala: 
"me ha pasado que no creen que soy colombiana, por mi fisonomía. Porque de pronto tienen la idea de que todos son negros". En el caso de los afrocolombianos/as, "la calle", y particularmente los medios de transporte, son indicados como lugares donde vivencian cotidianamente discriminaciones racistas. Leonardo (afrocolombiano, medio-bajo) indica: "Y en la calle siempre (discriminan) ¡ay!”; y Maricela (afrocolombiana, bajo) señala:

"Y acá es algo difícil, algunas personas no, no, no voy a decir todos, lo aceptan bien a uno... Otras, por lo menos en el metro, se han tapado la nariz, como si uno oliera feo. ¿Me entiende? 'Que esas negras tanto, ¿qué hacen aquí?'. Ahh y se me olvidaba, cuando nos tocaba trabajar en Nuble, nos tocaba tomar una micro (bus), la micro no nos paraba".

Una experiencia similar relata Yennifer (afrocolombiana, medio-bajo):

"Cuando tú pasas y una mujer te dice cosas, o como cuando tú vas en la micro y te miran así como de mala forma, o como que tú estás esperando la micro y si hay varios negros no te paran ... en ocasiones donde hemos estado varios morenos no para la micro".

Los espacios públicos como plazas o centros comerciales también son lugares donde los afrocolombianos han experimentado la discriminación. Así lo relata Marta (afrocolombiana, medio-bajo):

“...hace como un año, había un niño que se iba a caer de la escala en un centro comercial y yo lo cogí. Un niño lindo ¿no? blanco él, ojos verdes... yo lo cogí pa' salvarlo y la mamá 'no lo toque, no lo toque' y lo limpió ... ‘señora yo estaba salvado a su hijo' y todos 'sí lo estaba salvando, desagradecida' todo el mundo conmigo, y la señora también era rubia, alta... Entonces le limpió la mano, porque lo había tocado yo, lo limpió así 'ay no lo toque, no lo toque', 'no señora, no era por acariciarlo, no, sino que se iba a caer y si se cae al vacío se le mata el niño"”.

Evitar los espacios públicos y el transporte público es prácticamente imposible en una gran ciudad como Santiago. Sin embargo la hostilidad que vivencian algunos afrocolombianos hace que valoricen no tener que exponerse en estos sitios, como lo manifiesta Maricela (afrocolombiana, bajo): “...gracias a dios (para trabajar) aquí yo no tomo locomoción”. Esto puede tener consecuencias sobre su vida social en general y sobre las opciones laborales que busquen, ya que evitar los espacios públicos y el transporte colectivo en una ciudad de las dimensiones de Santiago limitará en fuerte medida las opciones laborales y de desarrollo social y cultural. 
Frente a estas situaciones de discriminación altamente violentas, los inmigrantes afrocolombianos desarrollan, en términos generales, dos estrategias. Por una parte, tratar de ser indiferentes, "no pescar", "no parar bolas" a los discriminadores. Esto es lo que señala Leonardo (afrocolombiano, medio-bajo):

"Yo no le replico a nadie por nada ... en Colombia yo era agresivo y era atrevido, y no, aquí no, aquí a mí me hablan y 'ahh... ah, no te pesco', sigo mi camino no más... Me da lo mismo, me da igual..."; Marta (afrocolombiana, medio-bajo) sigue la misma estrategia: "Pero yo no le paro bolas, yo cojo mi bolso y me voy, pesco mi bolso y me voy. Y así, estoy sola, luchando".

La otra estrategia es la reacción, responder al discriminador, incluso llegando a derivar en violencia física. Así lo señala Maricela (afrocolombiana, bajo):

"Discriminan. Nosotros les cocinamos, nosotras los atendemos, sale uno con una buena cara y ellos lo amargan al tiro a uno. Empiezan, por lo menos aquí un señor, 'deme una Coca-Cola negra', '¿Oiga va a empezar con su racismo?', ‘¡ahh!, iya te pusiste boba!, ándate... jándate a la chucha!’, me dijo. Y le dije: 'oiga me respeta, usted es bien atrevido', 'ah, te voy a acusar con tu jefa'. Le dije: '¿o sea que usted es el que me ofende y que me va a acusar ahí?, algún día nos encontramos por ahí y te voy a sacar la chucha', ijajaja!, vea, lo insulté y él me insultaba, pero no me dejé".

También sigue esta estrategia Diego (afrocolombiano, bajo): “...lastimosamente termina a veces en golpes pero ... si aquí alguien me dice, 'negro yo-no-sé-qué', yo no tengo ningún problema de levantarle la mano, pegarle, pa' que me respeten”.

De esta manera, los inmigrantes afrodescendientes deben enfrentar en múltiples ocasiones situaciones de discriminación en base al racismo. El color de piel se constituye así en una "racialización" (Stefoni, 2016) que marca profundamente las experiencias de los inmigrantes. Sin embargo, hay que mantener presente que, junto a ella, las diferenciaciones socioeconómicas también actúan estableciendo dinámicas disímiles según los estratos socioeconómicos. En relación a este punto es revelador el planteamiento de la única entrevistada afrodescendiente que pertenece a un estrato socioeconómico alto, ya que en su caso la condición de afrodescendiente no sería problema en sus relaciones cotidianas, pues no operaría la discriminación racial como opera en los casos de afrodescendientes de los estratos sociales más bajos. Yamilec (afrocolombiana, alto): 
"Conozco muchos colombianos que me hablan de discriminaciones, de situaciones difíciles, y no sé si es porque yo me muevo en un ambiente en que la gente es más culta que no me pasan estas cosas, ¿me entiendes? Entonces, a medida que tú tengas más cultura, más educación, es menos discriminación ... No es lo mismo pasar por una calle de Ñuñoa, que irse a una población, que te vean, y cuando te ven todos te quieren tocar, todos murmuran, te dicen cosas, porque ellos no han salido, no conocen, ¿sí me entiendes? Entonces, eso pasa”.

De esta manera, hay una interseccionalidad en la discriminación que incluye la variable racial y la variable socioeconómica. Las situaciones de maltrato, discriminación, xenofobia y estereotipos se concentran, y se dan con mayor intensidad, sobre el grupo de población afrodescendiente de estratos socioeconómicos bajos y medios-bajos.

\section{Conclusiones}

El colectivo de colombianos/as es una de las comunidades de inmigrantes más numerosas y de más rápido aumento en Chile (DEM, 2016), mostrando un gran crecimiento entre 2005 y 2015, tanto en los Permisos de Residencia Definitiva (más de dos mil por ciento en el periodo) como en las Visas Temporarias (1 940 por ciento). Además, la población colombiana residente en Chile posee diversidades que no son comunes en otros colectivos de inmigrantes, específicamente la heterogeneidad socioeconómica y la "racial". A través del procesamiento de la variable "profesión" de las bases de datos del DEM, se puede corroborar que los inmigrantes colombianos se distribuyen en diferentes estratos socioeconómicos, y si bien mayoritariamente pertenecen al estrato de bajos ingresos (poco menos de 50 por ciento según la aproximación realizada) — desempeñándose como garzones, obreros, asesoras del hogar, entre otros-, también un porcentaje significativo del colectivo colombiano pertenece a estratos medios (aproximadamente 30 por ciento) y altos (aproximadamente 20 por ciento) - contando entre ellos un importante número de profesionales como médicos, ingenieros y abogados-. Sobre la heterogeneidad racial no hay datos ni en registros, encuestas o censos, por lo que no podemos aproximarnos a la composición de la población sobre esta variable, sin embargo a través de las entrevistas se puede corroborar que hay un número significativo de colombianos y colombianas afrodescendientes, que pertenecen en su mayoría al estrato socioeconómico bajo.

La heterogeneidad interna de este colectivo hace que sea particularmente interesante estudiar su proceso de integración y las dificultades que enfrenta, por las distintas formas de discriminación que reciben según 
estratos socioeconómicos y "razas". La conclusión general es que efectivamente se producen diferencias importantes entre los distintos grupos socioeconómicos y "raciales" en su proceso de integración/exclusión y en las discriminaciones que sufren, particularmente en espacios como el laboral. En cuanto a los distintos grupos, las entrevistas muestran que los inmigrantes de estratos medios-altos y altos tienen mejores procesos de integración, mejores relaciones con los/as chilenos/as, y que las discriminaciones que reciben son de menor intensidad y frecuencia que los otros grupos. Los inmigrantes de estratos socioeconómicos medios-bajos y bajos enfrentan mayores problemas en sus procesos de integración y discriminaciones más frecuentes, aunque en general de baja intensidad. Sin embargo, hay importantes diferencias según las distintas "razas". Mientras los inmigrantes "blancos" o mestizos tienen experiencias de integración relativamente exitosas - aunque enfrentando más dificultades los inmigrantes de estratos bajos y medios-bajos que los de estratos altos y medios-altos-, los afrocolombianos/as relatan situaciones de discriminación cotidianas y de gran intensidad, que llegan a la violencia verbal, y en ocasiones a la física, afectando, en algunos casos seriamente sus procesos de integración a la sociedad, por ejemplo en el temor a utilizar medios de transporte urbanos o los espacios públicos, con los consiguientes problemas para acceder a trabajos, entre otros. Así, hay una diferencia radical en el trato que reciben las distintas "razas" de colombianos/as, ya que los afrocolombianos/as son víctimas de formas de discriminación de una intensidad mucho mayor que quienes no son afrodescendientes.

Es necesario interrogarse por las causas de estas conclusiones. Como se señaló en un inicio, Chile es un país con muy poca población afrodescendiente, y que sólo en los últimos años ha recibido inmigración de países como Colombia, Haití y República Dominicana, con una presencia significativa de afrodescendientes. Por ello, ni la cultura "nacional", ni las dinámicas sociales "nacionales", ni la historia "nacional", y tampoco el racismo "nacional" dirigido históricamente a personas de fenotipo "indígena", puede usarse para explicar que la población chilena tenga prejuicios racistas y trate de manera discriminatoria a las personas afrodescendientes, con las cuales ha tenido escasa relación. La explicación que se propone es que la cultura chilena expresa su participación en la geocultura colonialista y eurocéntrica del sistema-mundo capitalista, de la cual ha recibido una clasificación jerárquica de valorización de las "razas", en la cual la "raza negra" ocupa uno de los últimos eslabones de esta jerarquía racista mundial. 
En las últimas décadas la Perspectiva Decolonial ha planteado que es en el marco de la expansión intercontinental del sistema-mundo capitalista que se produce, desde su centro europeo, una geocultura que organiza las relaciones intersubjetivas entre los diferentes pueblos, culturas y "razas" que abarca este sistema. Es en el marco de esta geocultura que ha recorrido los más de quinientos años del sistema-mundo capitalista que debe insertarse la producción de las "culturas nacionales" como la chilena. No es sorprendente desde esta perspectiva que los patrones de integración, exclusión y discriminación que afectan a los diferentes colectivos de inmigrantes expresen la forma colonialista-eurocéntrica de la geocultura del sistema-mundo capitalista, al concentrar el comportamiento racista y xenofóbico sobre la población afrodescendiente. Este patrón es el que se está expresando en Chile hoy, es el que están reproduciendo en sus relaciones intersubjetivas con los inmigrantes los/as chilenos/as, incluso con aquellos colectivos de inmigrantes, como los afrodescendientes, que son desconocidos desde la experiencia nacional pero conocidos desde la experiencia geocultural del sistema-mundo, que los sitúa en los eslabones más bajos de la jerarquía racista-eurocéntrica definida por el colonialismo.

La posibilidad de que Chile se constituya en una sociedad multicultural, que pueda albergar una convivencia de calidad entre los múltiples colectivos que alberga y que sigue aumentando con la llegada de inmigrantes, donde no existan comunidades étnicas cerradas que sean objeto de discriminación racial y marginación socioeconómica, dependerá de una decidida acción desde políticas públicas basadas en derechos que busquen aminorar los efectos de la geocultura racista-colonialista en la que está inserto el país. Para ser exitosa, esta acción intercultural debe partir por constituir una clara conciencia del desafío que implica enfrentar, no unos determinados "prejuicios" que tiene una parte "racista" de la población, sino una geocultura de más de quinientos años que se ha producido y mantenido ya que se corresponde con un sistema de dominación y explotación a escala mundial. 


\section{REFERENCIAS BIBLIOGRÁFICAS}

Adorno, Theodor, 2001, "Sociedad", en Adorno, Theodor, Epistemología y Ciencias Sociales, Cátedra, Madrid.

Bonfil Batalla, Guillermo, 1987, México profundo. Una civilización negada, Grijalbo, México.

Cárdenas, Mauricio y Mejía, Carolina, 2006, Migraciones internacionales en Colombia: ¿qué sabemos?, en Working Paper Series, Documentos de Trabajo, núm. 30, fecha consulta 3/06/2016. Disponible en http://www.iadb.org/intal/intalcdi/ $\mathrm{PE} / 2009 / 02989 . \mathrm{pdf}$

Cardoso, Fernando Enrique y Faletto, Enzo, 1979, Dependencia y Desarrollo en América Latina: Ensayo de Interpretación Sociológica, Siglo XXI, México.

Castro-Gómez, Santiago, 2000, "Ciencias sociales, violencia epistémica y el problema de la "Invención del Otro", en Lander, Edgardo (compilador), La colonialidad del saber: eurocentrismo y ciencias sociales, CLACSO, Buenos Aires.

DEM, 2016, "Migración en Chile 2005-2014”, en Anuario Estadístico Nacional 2005-2014, Departamento de Extranjería y Migración del Ministerio del Interior y Seguridad Pública, fecha de consulta 23/01/2017. Disponible en http://www. extranjeria.gob.cl/media/2016/06/Anuario.pdf

Echeverri, María Margarita, 2016, "Otredad racializada en la migración forzada de afrocolombianos a Antofagasta (Chile)", en revista Nómadas, IESCO, Universidad Central, Bogotá.

El Mostrador, 2016, "Ossandón endurece su discurso hacia los extranjeros: Las puertas del país como las de la casa se abren pero no a todos", en El Mostrador, 28 de noviembre, sección Noticias, fecha de consulta 25/01/2017. Disponible en http://www.elmostrador.cl/noticias/pais/2016/11/28/ossandon-endurece-su-discurso-hacia-los-extranjeros-las-puertas-del-pais-como-las-de-la-casa-se-abrenpero-no-a-todos/

El Mostrador, 2017, "Inmigrantes: Piñera le quita el tema a Ossandón y logra sacar Bancard de la agenda", en El Mostrador, 29 de noviembre de 2016, sección Noticias, fecha de consulta 25/01/2017. Disponible en http://www.elmostrador. cl/noticias/pais/2016/11/29/inmigrantes-pinera-le-quita-el-tema-a-ossandon-ylogra-sacar-bancard-de-la-agenda/

Falquet, Jules, 2009, "Repensar las relaciones de sexo, clase y 'raza' en la mundialización neoliberal", en Dorlin, Elsa, Sexe, race, classe. Pour une épistémologie de la domination, PUF, Paris. Disponible en https://julesfalquet.files.wordpress. com/2010/05/repensar-todo-inedito.doc

Gissi, Nicolás, 2016, "Arraigo y desarraigo en los inmigrantes colombianos/as en Santiago de Chile. Incorporación social y transnacionalismo en el contexto de la globalización", en Aliaga, Felipe (editor), Migraciones internacionales. Alteridad y procesos sociopolíticos, Universidad Santo Tomás, Bogotá. 
GMG, 2013, "UN General Assembly high-level dialogue on international migration and development 2013", en Global Migration Group Position Paper, International Migration and Development, fecha consulta 25/07/2016. Disponible en http://www.globalmigrationgroup.org/sites/default/files/uploads/news/GMGposition-paper-on-2013-High-Level-Dialogue.pdf

González Casanova, Pablo, 2006, "Colonialismo interno (Una redefinición)”, en Borón, Atilio, Amadeo, Javier y González, Sabrina (compiladores), La teoría marxista hoy. Problemas y perspectivas, CLACSO, Buenos Aires.

Guibernau, Montserrat, 2009, La identidad de las naciones, Ariel, Barcelona.

INE, 2014, "I 'Encuesta de caracterización de la población afrodescendiente", en Informe de Resultados ENCAFRO 2013, fecha de consulta 23/01/2017. Instituto Nacional de Estadísticas, disponible en http:/www.ine.cl/canales/chile_estadistico/estadisticas_sociales_culturales/etnias/pdf/informe_de_resultados_encuesta_de_caracterizacion_de_la_poblacion_afrodescendiēnte_de_la_region_de_ arica_y_parinacota_2013.p̄ $\overline{\mathrm{d}} \mathrm{f}$

Kymlicka, Will, 1996, Ciudadanía multicultural, Paidós, Barcelona.

Lander, Edgardo, 2000, “Ciencias sociales, saberes coloniales y eurocéntricos”, en Lander, Edgardo (compilador), La colonialidaddel saber: eurocentrismo y ciencias sociales, CLACSO. Buenos Aires.

Martínez, Jorge y Orrego, Cristián, 2016, Nuevas tendencias y dinámicas migratorias en América Latina y el Caribe, en OIM y CEPAL, Serie Población y Desarrollo núm. 114, Naciones Unidas, Santiago de Chile.

Martínez, Jorge, 2011, Migración Internacional en América Latina y el Caribe. Nuevas tendencias, nuevos enfoques, Cepal, Santiago de Chile.

Mejía, William, 2012, "Colombia y las migraciones internacionales. Evolución reciente y panorama actual a partir de las cifras", en Revista Interdisciplinar da Mobilidade Humana vol. 20, núm. 39, pp. 185-210, Centro Scalabrianiano de Estudos Migratórios, Brasilia.

Mignolo, Walter, 2000, "La colonialidad a lo largo y a lo ancho: el hemisferio occidental en el horizonte colonial de la modernidad", en Lander, Edgardo (compilador), La colonialidad del saber: eurocentrismo y ciencias sociales, CLACSO, Buenos Aires.

OIM, 2015, "Los migrantes y las ciudades: Nuevas colaboraciones para gestionar la movilidad", en Informe sobre las migraciones en el mundo 2015, Organización Internacional para las Migraciones, fecha de consulta 17/06/2016. Disponible en http://publications.iom.int/system/files/wmr2015_sp.pdf

OIM, 2016, La Migración Colombiana en Argentina, Organización Internacional para las Migraciones, Amerian, Buenos Aires.

Palacios, Nicolás, 1987, Raza chilena, Antiyal, Santiago de Chile.

Pavez, Jorge, 2016, "Racismo de clase y racismo de género: "mujer chilena", "mestizo blanquecino" y "negra colombiana" en la ideología nacional chilena", en 
Tijoux, María Emilia, 2016, Racismo en Chile. La piel como marca de la inmigración, Editorial Universitaria, Santiago de Chile.

Portes, Alejandro, 2012, Sociología económica de las migraciones internacionales, Anthropos, Barcelona.

Restrepo, Eduardo y Rojas, Axel, 2010, inflexión decolonial: fuentes, conceptos y cuestionamientos, Universidad de Cauca, Popoyan.

Rojas, Mario, 2016, "El 52 por ciento de los imputados por delitos en el extremo norte del país son ciudadanos extranjeros", en El Mercurio 30 de agosto 2016, sección Nacional, fecha de consulta 25/01/2017. Disponible en http://impresa. elmercurio.com/Pages/NewsDetail.aspx?dt=2016-08-30\&dtB=09-04-2017\%20 0:00:00\&PaginaId $=6 \&$ bodyid $=3$

Rojas, Nicolás y Silva, Claudia, 2016, "La Migración en Chile: breve reporte y caracterización", en Informe OBIMID, julio/agosto 2016, fecha de consulta 04/12/2016. Disponible en http://www.extranjeria.gob.cl/media/2016/08/informe_julio_agosto_2016.pdf

Sassen, Saskia, 2015, Expulsiones. Brutalidad y complejidad en la economía global, Katz, Buenos Aires.

Solimano, Andrés, 2008, Migraciones internacionales en América Latina. Booms, crisis y desarrollo, FCE, México.

Stefoni, Carolina, 2016, "La nacionalidad y el color de piel en la racialización del extranjero. Migrantes como buenos trabajadores en el sector de la construcción", en Tijoux, María Emilia, Racismo en Chile. La piel como marca de la inmigración, Editorial Universitaria Santiago.

Tijoux, María Emilia, 2016, "Presentación”, en Tijoux, María Emilia, Racismo en Chile. La piel como marca de la inmigración, Editorial Universitaria Santiago.

Wallerstein, Immanuel, 2003, El moderno sistema mundial. La agricultura capitalista y los orígenes de la economía-mundo Europea en el siglo XVI, Siglo XXI, México.

Wallerstein, Immanuel, 2007, Geopolítica y geocultura. Ensayos sobre el moderno sistema mundial, Kairós, Barcelona. 


\section{RESUMEN CURRICULAR DE LOS AUTORES}

\section{Emiliano Gissi Barbieri}

Doctor en Antropología por la Universidad Nacional Autónoma de México (UNAM), Antropólogo Social de la Universidad de Chile y Magíster en Asentamientos Humanos y Medio Ambiente, en la Pontificia Universidad Católica de Chile. Académico del Departamento de Antropología y ex Jefe de Carrera de Antropología, Universidad de Chile. Dicta las cátedras de Antropología de la Migración, Antropología Económica y Cultura Latinoamericana. Es miembro del Claustro Académico del Programa de Magíster en Antropología Social y del Doctorado en Ciencias Sociales, de la Facultad de Ciencias Sociales, Universidad de Chile. Investigador Principal en Proyecto Fondecyt de Iniciación ( $\mathrm{N}^{\mathrm{o}}$ 11130287) "Migración y procesos de integración y exclusión social de colombianos y mexicanos en Chile. Estudio comparativo de dos casos de movilidad intra-latinoamericana" (2014-2016). Autor de un libro, varios capítulos de libros y de artículos publicados en revistas indexadas en Chile, México, Colombia y Argentina, sobre formas de integración y exclusión social de indígenas urbanos en Chile y México, así como de migrantes latinoamericanos en Chile. Dirección electrónica ngissi@uchile.cl

\section{Gonzalo Ghio Suárez}

Doctor en Ciencias Sociales por la Universidad de Chile, Sociólogo de la Universidad de Chile, Magister en Estudios internacionales de la Universidad de Chile y postgraduado en Análisis Demográfico en CELADECEPAL. Fue investigador del Departamento de Demografía del Instituto Nacional de Estadísticas donde trabajó en el desarrollo de metodologías de recolección de información de migraciones internas e internacionales, en el análisis de las tendencias demográficas para la definición de proyecciones de población, entre otros temas. Actualmente desarrolla su investigación de tesis doctoral en el estudio de las actuales dinámicas de expansión del sistema capitalista mundial, y su impacto en los espacios sociales y poblaciones de la periferia.

Dirección electrónica gonzaloghio@gmail.com

Artículo recibido el 12 de abril de 2017 y aprobado el 28 de julio de 2017. 